\title{
EFFECT OF YOHIMBINE ON CLOMIPRAMINE-INDUCED SEXUAL DYSFUNCTION IN MALE RATS
}

\author{
SHESHADRI SHEKAR*, MOHAMMED VAZIR, BINDHU KEMPANNA, SHIVA KUMAR MIRYALA, ASHOK SUBEDI
}

Department of Pharmacology, Hillside College of Pharmacy and Research Centre, Bengaluru, Karnataka, India. Email: Sheshadrishekar@gmail.Com

Received: 20 May 2016, Revised and Accepted: 05 November 2016

ABSTRACT

Object: The present investigation has been carried out to find out the effect of yohimbine on clomipramine-induced sexual dysfunction in male rats.

Methods: The male rats were treated with clomipramine and yohimbine simultaneously for 60 days. During the treatment, all the male rats were challenged with the female rats which are in estrous phase and their sexual behavior was observed under dim red light. Half of the animals in each group and remaining on $60^{\text {th }}$ day were sacrificed, blood was collected and serum separated. Testis was collected and preserved in $10 \%$ formalin for subsequent histopathological examination.

Results: The study reveals that yohimbine failed to antagonize the clomipramine-induced sexual dysfunction in male rats in all aspects, except the partial improvement in the sexual behavior.

Conclusion: Yohimbine a well-known aphrodisiac failed to antagonize the clomipramine-induced sexual dysfunction in male rats. The decrease in testosterone levels, a decrease in spermatozoa count were continued even in the presence of yohimbine except improvement in the sexual behavior parameters. Hence, yohimbine could not be a safe antidote against clomipramine-induced sexual dysfunction in male rats.

Keywords: Yohimbine, Clomipramine, Testosterone, Male rat sexual competence, Testicular damage.

(c) 2017 The Authors. Published by Innovare Academic Sciences Pvt Ltd. This is an open access article under the CC BY license (http://creativecommons. org/licenses/by/4. 0/) DOI: http://dx.doi.org/10.22159/ajpcr.2017.v10i2.12970

\section{INTRODUCTION}

Psychic depression is a disorder that requires treatment for longer periods. It is known to be precipitated because of excessive metabolism of centrally located monoamines, particularly serotonin. Hence, monoamine oxidase inhibitors, tricyclic antidepressants, and specific serotonin inhibitors (SSRI) are used for its treatment. The antidepressants elevate the monoamines, particularly serotonin for its effect in the treatment of depression. In doing so, they produce sexual dysfunction as a rise in central serotonin leads to decreased libido. Clomipramine (Clmp) is the imipramine analog of chlorpromazine. Due to its action against anxiety disorders and panic attacks, it is the only drug with 2 entries in the essential drugs list of the WHO. Regarding the compulsive disorders, it is now the "gold standard" of therapy against which other drugs are measured [1,2]. In addition to SSRI, action clomipramine can block adrenergic, cholinergic, and dopaminergic receptors which also contribute for sexual dysfunction [3]. It was shown that $96 \%$ of males and females on clomipramine suffered from delayed orgasm representing the highest rate of antidepressantinduced sexual dysfunction with any medication and it was consistent with clomipramine's potent serotonergic activity [4,5].

The sexual function involves central, and peripheral neuronal activity, hormonal activity, and peripheral cellular activity. Compared to other tricyclic antidepressants, it has a greater effect on dopamine blockade and serotonin reuptake inhibition [6]. These implicate for prolactin release [7] and orgasmic dysfunction mediated through $5-\mathrm{HT}_{2}$ receptors [8]. Moreover, peripheral antimuscarinic and $\alpha$ adrenergic blockade [9-12], effects have been implicated in the clomipramineinduced sexual dysfunction. It was also reported in our studies that clmp dose-and time-dependently decreased the testosterone levels, sexual competence of male rats and damaged the testes [13]. In another study, amantadine failed to antagonize Clomipramine-induced sexual dysfunction in male rats [14]. Various treatment strategies exist for antidepressant-induced sexual dysfunction. They are (1) try waiting (which does not really work)for spontaneous remission of symptoms as tolerance to the drug may develop, (2) decreasing the dose of the current antidepressant or (3) switching to a different antidepressant [15]. Although sometimes effective these strategies scare patients as switching to a different antidepressant that works in one may not work as well in another. The decrease in the dose of the current drug and/or taking a drug holiday (Purposely skipping medication for a period when sexual activity is anticipated) may cause a relapse of depression. The prevention of the relapse/worsening of depression are of utmost importance to the patient even more so than a healthy sex life. Therefore, the most promising treatment is to stick to the current effective antidepressant and add another medication (or antidote) by a trial and error method to suppress sexual side effects. However, no perfect solution exists to date as these antidotes have their own adverse effects, and hence the search for proper medication be continued [16].

The drug yohimbine is known as adrenergic $\alpha 2$ receptor blocker and as an aphrodisiac since a long time. Some of the textbooks in pharmacology mention that it is claimed as aphrodisiac without scientific support. The $\alpha 2$ activity being associated with autoregulation of noradrenaline, its blockade led to increased secretion and increased the level of noradrenaline which is known to be associated with improved sexual function. The recent literature reports on yohimbine indicate that it improved erectile dysfunction in aged male rats [17]. Other reports indicated that it improved sexual function in male rats mostly through dopamine-related activity rather than $\alpha 2$ adrenergic blocking activity which improves central adrenergic activity. The net result of the recent reports was that it improves sexual function and as per one report, it improves sexual function even in sexually satiated rats [18].

As per the literature, yohimbine improves sexual activity by involving noradrenaline and dopamine of central origin and improves sexual behavioral parameters [19]. However, its activity on the hormonal 
and histological parameters of testes is not known. An aphrodisiac can be clinically well-accepted if it works well centrally and peripherally. Hence, to know more about the influence of yohimbine on sexual activity on clomipramine-induced sexual dysfunction the present study was undertaken.

\section{METHODS}

- Yohimbine Hcl: John Baker Inc., Colorado, U.S.A

- Clomipramine Hcl: Psychotropic India Ltd, Ghaziabad

- Carboxymethyl cellulose: Nice chemicals, Cochin

- Diethyl ether: Nice chemicals, Cochin

- Estradiol benzoate: Sigma-Aldrich, U.S.A

- Eosin: Nice chemicals, Cochin

- Hematoxylin: Nice chemicals, Cochin

- Progesterone: Glen mark, Mumbai

- Sesame oil: N. Ravindra Company, Mumbai

- $\quad$ Rat feed: Saidurga animal feeds, Bengaluru

- Testosterone and prolactin kits: DPC, New York, USA

\section{Preparation of drug solution}

Clomipramine solution

The maximum human therapeutic dose of clomipramine was $300 \mathrm{mg} /$ daily. From this $1 / 2$ therapeutic dose and therapeutic dose were calculated based on body surface area and was found to be $13.5 \mathrm{mg} / \mathrm{kg}$ and $27 \mathrm{mg} / \mathrm{kg}$ [20]. The control group received vehicle. Yohimbine dose used was as per the earlier studies $2 \mathrm{mg} / \mathrm{kg}$.

\section{Animal preparation}

A total of 36 male and 36 female Sprauge-Dawley albino rats were purchased from central animal house NIMHANS, Bengaluru. All animals were housed in a group of four males and females separately in plexiglass cages $(62 \times 40 \times 21)$ in an acclimatized colony room $\left(25 \pm 0.5^{\circ} \mathrm{C}\right)$ maintained on a $12 / 12 \mathrm{hrs}$ light/dark cycle. The rats were 4 months old. They were weighed around 200-300 g each and females 250-350 g each. They were fed on commercial pellet feed and water was available ad libitum. Prior approval was obtained from the institutional ethical committee for conducting the studies.

\section{Animal treatment}

The male rats were randomly divided into three groups of 12 rats in each. Group I served as control, Groups II and III were treated with yohimbine $(2 \mathrm{mg} / \mathrm{kg}$ ) orally, 30 minutes later they were treated with clomipramine $13.5 \mathrm{mg} / \mathrm{kg}$ and $27 \mathrm{mg} / \mathrm{Kg}$ body weight, respectively, for 60 days. The control group received vehicle. All the drugs were given by oral route.

\section{Procedure for overiectomization}

Ovariectomy was necessary to make the female non-pregnant when mated with male rats and to reuse them repeatedly during the period of experimentation [21]. To bring them to oestrous phase, they were given $12 \mu \mathrm{g} /$ rat of estrodiol benzoate (sesame oil) S.C. 56 hrs before and $500 \mu \mathrm{g}$ (sesame oil) of progesterone 4-6 hrs before the copulatory test [22].

The drugs were administered orally to male rats and the animals were observed for their sexual behavior. Female rats were prepared for sexual receptivity as described earlier. Male rat sexual behavior was studied as explained elsewhere [23]. The drugs were administered orally to male rats and the animals were observed for their sexual behavior. Female rats were prepared for sexual receptivity as described earlier. Then, the highly receptive female was introduced into the male's cage a rectangular glass observation cage $(62 \mathrm{~cm} \times 40 \mathrm{~cm} \times 21 \mathrm{~cm})$ in which male acclimatizes for 2 hrs. They were observed in the cage for 30 minutes under dim red light. When there was no mount latency, intromission, ejaculation, and post ejaculation pause their total latent period was taken as 1800 seconds. Tests were terminated immediately after the first post-ejaculatory intromission/mount.

\section{Collection of blood sample and testes}

Half of the animals in each group on the $30^{\text {th }}$ day and remaining on the $60^{\text {th }}$ day were sacrificed for blood sample collection and histopathological examination of testes. Blood was collected through cardiac puncture using a 16 no needle under mild ether anesthesia and allowed to settle for some time. After centrifugation serum was separated and stored at $-20^{\circ}$ for subsequent hormonal estimation. Testes were also collected and processed for the histopathological studies [24].

\section{RESULTS}

1. Influence of yohimbine on clomipramine-induced decline in sexual competence in male rats

Yohimbine significantly increased the number of rats intromitting and ejaculating in both $1 / 2$ TD and TD of clmp.

A significant decrease in mount latency, intromission latency, ejaculation latency, post ejaculation pause was observed. Increase in the copulating efficiency and decrease the inter-copulating interval was observed at both doses of clmp.

Hence, to conclude yohimbine significantly retained the sexual behavior of male rats treated with clomipramine, but still failed to maintain the normal sexual competence.

Results indicate that the number of rats mounted, intromitted, and ejaculated was significantly more in combination with clomipramine. There was a decrease in the inter-copulatory interval and increased copulatory efficiency. The number of mounts, number of intromissions and ejaculations were more than that of the clomipramine alone treated groups. A significant decrease in the post-ejaculatory interval was also observed. However, yohimbine also failed to prevent the sexual dysfunction completely in the male rats treated with clomipramine. Results were tabulated in Table 1.

2. Influence of yohimbine and clomipramine on serum testosterone At the end of 30 and 60 days treatment, no improvement in the testosterone level was observed. Yohimbine failed to antagonize clmp induced decline in serum testosterone levels. The results were tabulated in Table 2.

3. Histological studies of testes

The decrease in the sperm cells counted namely spermatogonia, preleptotene, pachytene, and secondary spermatocytes was continued with the yohimbine $+27 \mathrm{mg} / \mathrm{kg}$ clmp treatment at the end of 30 days and 60 days indicating that yohimbine did not prevent the testes from the damage induced by clmp. Results were tabulated in Tables 3 and 4 testicular damage was shown in Figs. 1-5.

\section{DISCUSSION}

The sexual response is the result of neuronal (both central and peripheral), hormonal and peripheral activities. The central activity is influenced by several mediators, the notable among them being noradrenaline, dopamine, and serotonin.

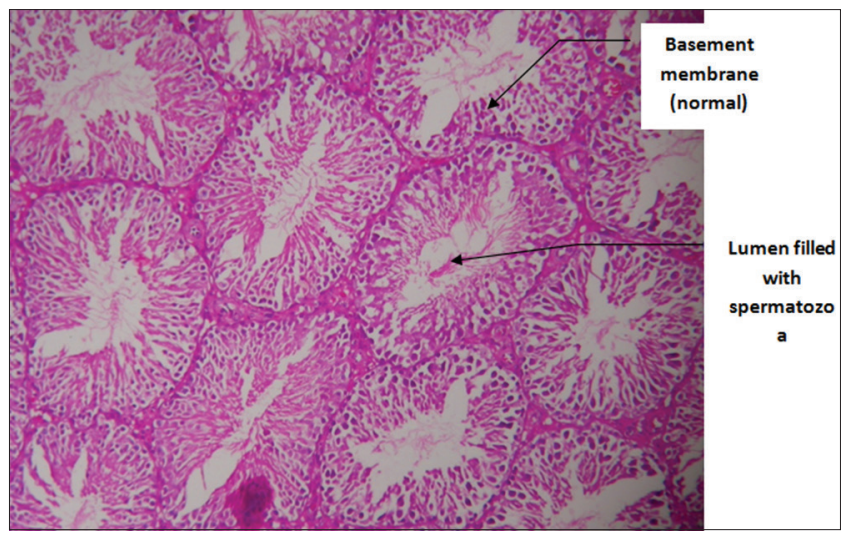

Fig. 1: Cross section of seminiferous tubules. Control $\times 400$ 




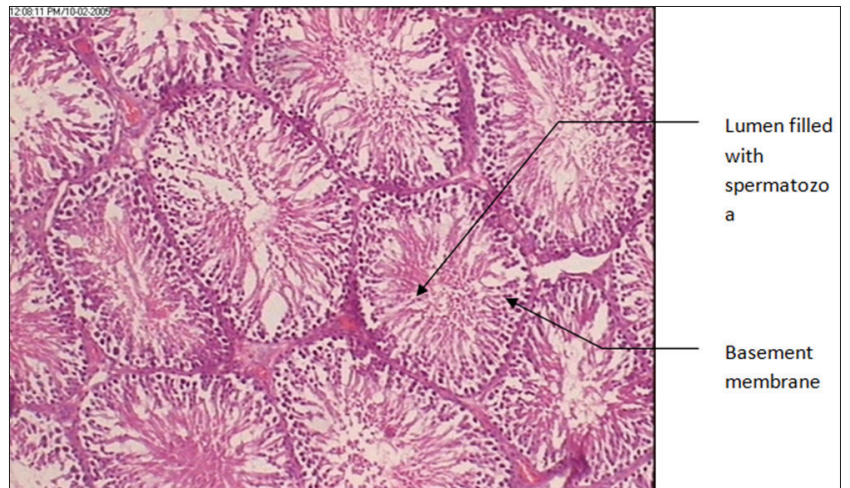

Fig. 2: Cross section of seminiferous tubules. Yohimbine + clomipramine $13.5 \mathrm{mg} / \mathrm{kg} 30$ days treatment $\times 400$

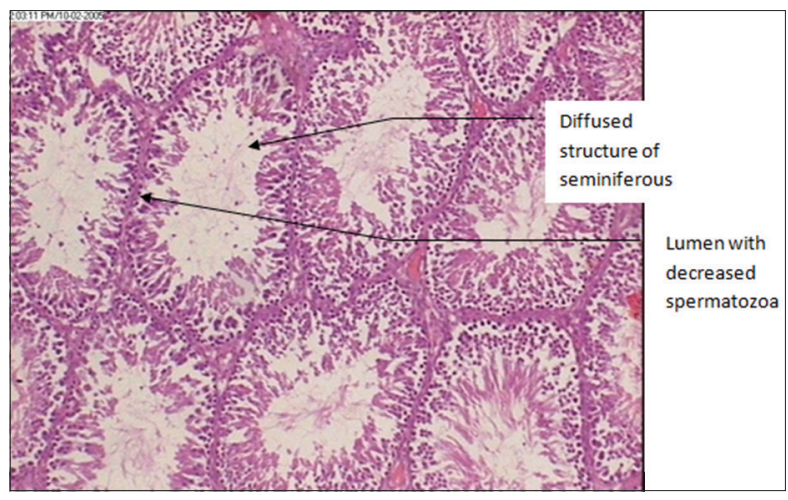

Fig. 3:

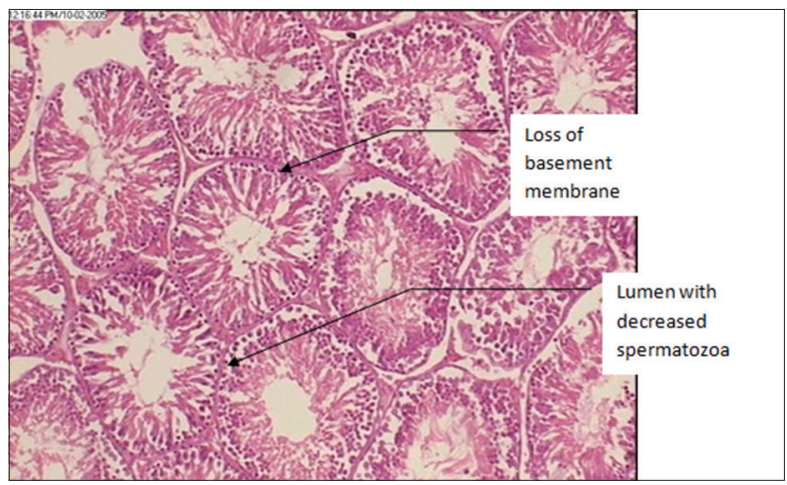

Fig. 4: Cross section of seminiferous tubules. Yohimbine + clomipramine $27 \mathrm{mg} / \mathrm{kg} 30$ days treatment

In the CNS, antagonism of adrenergic $\alpha 2$ receptors by other drugs appears to be the most probable mechanism for the important role of norephinephrine (NE) in enhancing sexual functions especially arousal $[25,26]$. Direct administration of the $\alpha 2$ agonist clonidine to the median preoptic area in male rats demonstrated increased latency to ejaculate and longer inter-couplatory interval. Clonidine action could be reversed by yohimbine a $\alpha 2$ antagonist. $\alpha 2$ antagonism can be understood as blocking the inhibitory activities of autoreceptors, leading to increased noradrenaline transmission resulting in C N S prosexual effects [27].

Blocking catecholamine (CA) synthesis with the CA synthesis inhibitor $\alpha$ - methylP-tyrosine, markedly reduced masculine sexual behavior pointed to a fundamental role of the catecholaminergic system in the expression of sexual behavior [28].

Guanethidine, an adrenergic neuronal blocking agent, exerts its antihypertensive effect by preventing the intraneuronal storage 
Table 2: Influence of yohimbine and clomipramine ( 30 days and 60 days) on serum testosterone (Data given as mean+SEM, $n=6$ )

\begin{tabular}{lll}
\hline Treatment & Testosterone $\mathbf{~ g} / \mathbf{m l}$ after 30 days & Testosterone ng/ml after 60 days \\
\hline Control $1 \mathrm{ml} / \mathrm{kg}$ CMC suspension & $6.55 \pm 0.66$ & $5.07 \pm 0.3$ \\
Yohimbine + clomipramine $13.5 \mathrm{mg} / \mathrm{kg}$ & $8.57 \pm 0.39$ & $8.5 \pm 0.65$ \\
Yohimbine + clomipramine $27 \mathrm{mg} / \mathrm{kg}$ & $3.27 \pm 0.351^{* *}$ & $2.5 \pm 0.52^{* *}$ \\
\hline
\end{tabular}

Significant at $\mathrm{p}<0.05^{*}, 0.01^{* *}$ compared to control (student $t$-test), CMC: Carboxy methyl cellulose

Table 3: Yohimbine and clomipramine treatment (30 days) influence on histology of testes (data given as mean+SEM $n=6$ )

\begin{tabular}{|c|c|c|c|c|c|}
\hline Treatment ( 30 days) & Sertoli cells & SP Gonia & Preleptotene & Pachytene & S.spermotocytes \\
\hline Control $1 \mathrm{ml} / \mathrm{kg}$ CMC suspension & $3.5 \pm 0.288$ & $8.25 \pm 0.4$ & $21.25 \pm 0.8$ & $27 \pm 0.4$ & $48.75 \pm 0.75$ \\
\hline Yohimbine + clomipramine $13.5 \mathrm{mg} / \mathrm{kg}$ & $3.25 \pm 0.25$ & $8.25 \pm 0.47$ & $22.7 \pm 0.4$ & $29.5 \pm 1.19$ & $54.5 \pm 1.25$ \\
\hline Yohimbine + clomipramine $27 \mathrm{mg} / \mathrm{kg}$ & $2.5 \pm 0.28$ & $6 \pm 0.4^{* *}$ & $17.25 \pm 0.7^{* *}$ & $23.5 \pm 0.40^{* *}$ & $37.5 \pm 1.56^{* *}$ \\
\hline
\end{tabular}

Significant at $\mathrm{p}<0.05^{*}, 0.01^{* *}$ compared to control (student $t$-test), CMC: Carboxy methyl cellulose

Table 4: Yohimbine and clomipramine treatment (60 days) influence on histology of testes (data given as mean+SEM $n=6$ )

\begin{tabular}{llllll}
\hline Treatment (30 days) & Sertoli cells & SP Gonia & Preleptotene & Pachytene & Secondary spermatocytes \\
\hline Control & $3.5 \pm 0.288$ & $8.25 \pm 0.4$ & $21.25 \pm 0.8$ & $27 \pm 0.4$ & $48.75 \pm 0.75$ \\
Yohimbine + clomipramine $13.5 \mathrm{mg} / \mathrm{kg}$ & $3 \pm 0.4$ & $7.5 \pm 0.28$ & $23 \pm 0.8$ & $28 \pm 1.08$ & $54.75 \pm 2.4$ \\
Yohimbine + clomipramine $27 \mathrm{mg} / \mathrm{kg}$ & $3 \pm 0.2$ & $7.5 \pm 0.2$ & $16 \pm 0.4^{* *}$ & $22.75 \pm 0.80^{* *}$ & $31.25 \pm 0.8^{* *}$ \\
\hline
\end{tabular}

Significant at $\mathrm{p}<0.05^{*}, 0.01^{* *}$ compared to control (student $t$-test)

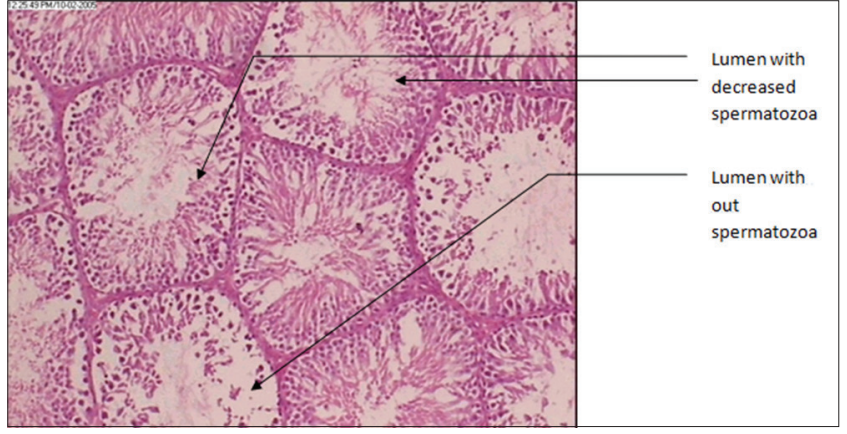

Fig. 5: Cross section of seminiferous tubules. Yohimbine + clomipramine $27 \mathrm{mg} / \mathrm{kg} 60$ days treatment

granule reuptake of NE, thereby blocking sympathetic neuronal activity. Methyldopa, a centrally acting sympatholytic agent, is metabolized to the false neurotransmitter $\alpha$ methyl NE. $\alpha$ methyl NE decreases the sympathetic outflow from the brain by competing with NE in sympathetic axons and by stimulating $\alpha$ receptor sites in the CNS. The common side effects of methyldopa are impotence, decreased libido, ejaculatory difficulties, and gynecomastia [29]. Since yohimbine is known to influence adrenergic function by blockade of $\alpha 2$ adrenergic receptors and is claimed to be aphrodisiac, its activity on the sexual function was evaluated in the present model in the presence of clomipramine.

The observed improvement in the male rat sexual competence may be due to its action on both the noradrenergic and dopaminergic system. It increases the turnover of rat cerebral noradrenaline, through the interaction with $\alpha 2$ adrenoreceptors [30]. The alkaloid also increases brain dopamine turnover an effect attributed to an indirect action of the drug on dopaminergic neurons exerted through changes in noradrenaline transmission [31]. The same effect of yohimbine has been observed in mice and was proposed to be exerted by the selective blockade of $\alpha 2$ autoreceptors [32].

Regarding the noradrenergic system, it was reported that yohimbine significantly increased the proportion of sexually exhausted rats showing mating behavior $24 \mathrm{hrs}$ after the copulation to exhaustion session [33]. In addition, it was reported that the neurotoxic lesion of the noradrenergic system blocks the ability of the $5-\mathrm{HT} 1_{\mathrm{A}}$ agonist 8 - hydroxyl - 2- (Di- n. propylamine) tetralin (8 - OH DPAT) and the $\mu$ and $\delta$ opioid receptor antagonist, naloxone to induce mating behavior in sexually satiated rats [34]. These findings suggest that the integrity of the noradrenergic system is essential for the pharmacological establishment of copulatory behavior. The central and peripheral effects of yohimbine+clomipramine associated with sexual function were given in Table 1 and represented graphically in.

Tonic adrenergic tone at $\alpha 1$ and $\alpha 2$ receptors act to keep the penis in a flaccid state and $\alpha 1$ blockers can produce prolonged erection and priapism [35]. This erection preventing norepinephrine-induced penile vascular smooth muscle contractility is blocked by either $\alpha 1$ antagonist, for example, prazocin or $\alpha 2$ antagonist yohimbine [36,37]. By a mechanism not clear and presumably under the influence of nitric oxide and vasoactive intestinal polypeptide (VIP). Adrenergic stimulation ceases to be inhibitory after sexual stimulation and was associated with increasing arousal in both men and women [38].

In our experiments, change in sexual behavior parameters was observed without change in the hormonal (testosterone), histological parameters (testicular) in the presence of the treatment with yohimbine. It clearly showed that it affected the adrenergic $\alpha 2$ receptors and its related activities also. It is well-established that $\alpha 2$ receptor blocking activity is associated with central and peripheral noradrenergic activity and also with associated dopamine function, both leading to behavioral sexual improvement. The results also indicated that yohimbine does not influence the testosterone or the peripheral histological parameters and its main action was due to its influence on adrenergic and associated dopaminergic system. Yohimbine, an antagonist at inhibitory noradrenergic $\alpha 2$ receptors, has been used to increase the limbic levels of norepinephrine. Yohimbine also increases parasympathetic activity $[39,40]$.

Ultimately yohimbine appears to improve sexual behavior through its $\alpha 2$ - blockade action which is reported to be associated with increased central and peripheral noradrenergic, dopaminergic functions and peripheral parasympathetic functions through NO and VIP.

The study indicated that yohimbine can be a supportive drug to antagonize sexual dysfunction produced by antidepressant clomipramine. It cannot be used alone to totally antagonize the sexual dysfunction of clomipramine as it did not prevent the hormonal and cellular changes. 


\section{REFERENCES}

1. Rothschild AJ. New directions in the treatment of antidepressant-induced sexual dysfunction. Clin Ther. 2000;22 Suppl A: A42-57.

2. Baldwin DS. Psychotropic drugs and sexual dysfunction. Rev Psychiatry 1995;7:262-73.

3. Bernik M, Vieira AH, Nunes PV. Bethanecol chloride for treatment of clomipramine-induced orgasmic dysfunction in males. Rev Hosp Clin Fac Med Sao Paulo 2004;59(6):357-60.

4. Monteiro WO, Noshirvani HF, Marks IM, Lelliott PT. Anorgasmia from clomipramine in obsessive-compulsive disorder. A controlled trial. $\mathrm{Br} \mathrm{J}$ Psychiatry 1987;151:107-12.

5. Fava M, Ramkin M. Sexual dysfunction and SSRIs. J Clin Psychiatry 2002;63 Suppl 5:13-6

6. Blackwell B. Antidepressant drugs. In: Dukes MN Meyer's Side Effects of Drugs. Amsterdam: Elsevier; 1984. p. 24-61.

7. Jones RB, Luscombe DK, Groom GV. Plasma prolactin concentrations in normal subjects and depressive patients following oral clomipramine. Postgrad Med J 1977;53 Suppl 4:166-71.

8. Pomerantz SM, Hepner BC, Wertz JM. Serotonergic influences on male sexual behavior of rhesus monkeys: Effects of serotonin agonists. Psychopharmacology (Berl) 1993;111(1):47-54

9. Sorscher SM, Dilsaver SC. Antidepressant-induced sexual dysfunction in men: Due to cholinergic blockade? J Clin Psychopharmacol 1986;6(1):53-5.

10. Beaumont G, Mayes A. Do task and sex differences influence the visual evoked potential? Psychophysiology 1977;14(6):545-50.

11. Aizenberg D, Zemishlany Z, Hermesh H, Karp L, Weizman A. Painful ejaculation associated with antidepressants in four patients. J Clin Psychiatry 1991;52(11):461-3.

12. Balon R, Yeragani VK, Pohl R, Ramesh C. Sexual dysfunction during antidepressant treatment. J Clin Psychiatry 1993;54(6):209-12.

13. Devang SS, Satyanarayana S, Kumar E, Vivek B. Clomipramine affects sexual behaviour and reproductive functions in male rats. Int $\mathrm{J}$ Health Sci 2010;3(3):341-7.

14. Devaangam SS, Satyanarayana S, Kumar KE, Vivek B, Velmurugan C, Kumar A. Amantadine failed to antagonize the clomipramine induced sexual dysfunction in male rats. Oman Med J 2011;26(6):404-9.

15. Gitlin MJ. Effects of depression and antidepressants on sexual functioning. Bull Menninger Clin 1995;59(2):232-48.

16. Lane RM. A critical review of selective serotonin reuptake inhibitor-related sexual dysfunction; Incidence, possible aetiology and implications for management. J Psychopharmacol 1997;11(1):72-82.

17. Smith ER, Davidson JM. Yohimbine attenuates aging-induced sexual deficiencies in male rats. Physiol Behav 1990;47(4):631-4.

18. Rodríguez-Manzo G. Yohimbine interacts with the dopaminergic system to reverse sexual satiation: Further evidence for a role of sexual motivation in sexual exhaustion. Eur J Pharmacol 1999;372(1):1-8.

19. Price J, Grunhaus LJ. Treatment of clomipramine-induced anorgasmia with yohimbine: A case report. J Clin Psychiatry 1990;51(1):32-3.

20. Paget GE, Barner JM. In: Laurence DR, Bacharach AL, editors. Evaluation of Drug Activities: Pharmacometrics. Vol. 1. New York: Academic Press; 1964.

21. Agmo A. Male rat sexual behavior. Brain Res Brain Res Protoc 1997;1(2):203-9
22. Amin KM, Khan MN, Rahman SZ, Khan NA. Sexual function improving effect of Mucuna puriens in sexually normal male rats. Phytotherapia 1996;67:53-8.

23. Ratnasooriya WD, Jayakody JR, Dharmasiri MG. Sodium nitroprusside impairs sexual competence of male rats. Hum Exp Toxicol 2004;23(4):187-92.

24. Culling CF, Rath MR. FIMLS Vancover Canada. Cellular Pathology Technique. Vol. 4. London: Butterworth Publisher; 1976. p. 29-138.

25. Bancroft J, Munoz M, Beard M, Shapiro C. The effect of a new alpha-2 adrenoceptor antagonist on sleep and nocturnal penile tumescence in normal male volunteers and men with erectile dysfunction. Psychosom Med 1995;57(4):345-56.

26. Guiliano F, Rampin O. Central adrenergic control of penile erection. Int J Impot Res 2000;12 Suppl: S13-9.

27. Meston CM, Gorzalka BB, Wright JM. Inhibition of subjective and physiological sexual arousal in women by clonidine. Psychosom Med 1997;59(4):399-407.

28. Wheatley D. Psychopharmacology and Sexual Disorders. Oxford: Oxford University Press; 1983. p. 1-7.

29. Holland OB, Fairchild C, Gomez-Sanchez GE. Effect of guan Benz and hydrochlorothiazide on blood pressure and plasma renin activity. J Clin Pharmacol 1981;21:133.

30. Anden NE, Strombom U. Adregenic receptor blocking agents: Effects on central noradrenaline and dopamine receptors and on motor activity. Psychopharmacologia 1974;38(2):91-103.

31. Andén N, Grabowska M. Pharmacological evidence for a stimulation of dopamine neurons by noradrenaline neurons in the brain. Eur $\mathrm{J}$ Pharmacol 1976;39(2):275-82.

32. Andén NE, Pauksens K, Svensson K. Selective blockade of brain alpha 2-autoreceptors by yohimbine: Effects on motor activity and on turnover of noradrenaline and dopamine. J Neural Transm 1982;55(2):111-20.

33. Rodríguez-Manzo G, Fernández-Guasti A. Reversal of sexual exhaustion by serotonergic and noradrenergic agents. Behav Brain Res 1994;62(2):127-34.

34. Rodriguez-Manzo G, Fernández-Guasti A. Participation of the central noradrenergic system in the re establishment of copulatory behaviour of sexually exhausted rats by yohimbine, Naloxone and 8-OH-DPAT. Brain Res Bull 1995;38:399-404.

35. Creed KE, Carati CJ, Keogh EJ. The physiology of penile erection. Oxf Rev Reprod Biol 1991;13:73-95.

36. Kirkeby HJ, Forman A, Sørensen S, Andersson KE. Alpha-adrenoceptor function in isolated penile circumflex veins from potent and impotent men. J Urol 1989;142(5):1369-71.

37. Bancroft J. Effects of alpha-2-blockade on sexual response: Experimental studies with delaquamine (RS15385). Int J Impot Res 2000;12 Suppl 1:S64-9.

38. Meston CM, Frohlich PF. The neurobiology of sexual function. Arch Gen Psychiatry 2000;57(11):1012-30.

39. Medical Economics. Physicians' Desk Reference (PDR). 55 $5^{\text {th }}$ ed. Montvale NJ: Medical Economics; 2001.

40. Vogt HJ, Brandl P, Kockott G, Schmitz JR, Wiegand MH, Schadrack J, et al. Double-blind, placebo-controlled safety and efficacy trial with yohimbine hydrochloride in the treatment of nonorganic erectile dysfunction. Int J Impot Res 1997;9(3):155-61 